\title{
Editorial: \\ A Manual for Journal of Nanophotonics Authors
}

\author{
Akhlesh Lakhtakia \\ Editor-in-Chief
}

Over the last twelve months, I have examined every article submitted for possible publication in the Journal of Nanophotonics. Several issues have reared their heads repeatedly, which prompted me to write a simple manual for prospective authors.

Choose the right size. Should you write a letter or a full paper? Your choice depends only partly on the contents of the intended paper. Letters are appropriate to present either work of momentous importance that must be published quickly, or incidental results that would add to the literature but are not of sufficient importance to merit publication as a full paper.

A full paper contains a lot of necessary detail. Only a small fraction of researchers will be highly interested in knowing all the details. Many full papers are read carefully only by graduate students. Make sure that you provide enough details so that your reader is not frustrated.

Review the literature. Before writing your manuscript, spend some time reviewing the relevant literature. You have to create a context for your work. Context is not created simply by citing many papers. Instead, think of a story about your work to tell your fellow researchers. Tell that story as the introductory part of your manuscript. The papers that appear in that story must be cited.

Learn to write well. Perhaps, there is really only one effective way to learn to write well: by reading. Good writers are voracious readers. Memorizing rules may help you, but you will instinctively write well after internalizing enough examples of good writing.

If you have not done so already, read a good book on writing a composition that is not literary. The following books are strongly recommended: (i) E. Bender, The Most Common Errors In English Usage And How To Avoid Them, (ii) W. Strunk, Jr., and E. B. White, The Elements of Style, and (iii) E. Gowers, The Complete Plain Words. A sturdy dictionary of English usage is well worth the investment. Read such books often, even if your native language is English.

Write an interesting manuscript. Your manuscript must report novel research, to the best of your knowledge. The parts of a paper that attract a reader most are its title, abstract, introduction, and the concluding section. If the title is not interesting and informative, potential readers will not bother to read even the abstract. The most that you can hope for is that the abstract will be widely read. If it is sufficiently inviting, a reader may then scan the paper for figures and maybe read the concluding section.

The following tips will help you write a manuscript that editors, reviewers and readers will find exciting:

- Choose a descriptive, attractive, and short title for the manuscript. This task requires some thought, as all three requirements should be met. The title should be chosen after finishing the rest of the manuscript.

- The abstract should also be written last. An abstract is a miniature stand-alone paper that should convey the most important details of the work as well as the chief results 
without reference to the rest of the manuscript. An abstract must not be written as an advertisement of the manuscript. Avoid the phrase "In this paper". Instead of "is studied/examined/presented/investigated," use "was studied/examined/presented/ investigated".

- The introduction must contextualize the research being reported. Review the literature, as advised earlier, and then create a context.

- Avoid the temptation to recount the history of science in your manuscript, unless you can write with the same accuracy as a historian of science.

- Break up your manuscript into sections and subsections. Every section and subsection must have a transparent theme. Avoid single-sentence paragraphs.

- The concluding section should not merely recount the work you did and the results that you reported in the previous sections. The concluding section should contain specific and broad conclusions that could be distilled from the results in the previous sections. In addition, do indulge in some speculation as to the implications of those conclusions.

- Avoid excessive use of jargon. Precision is important for scientific communication, but too much of jargon restricts the intelligibility of your manuscript to only a tiny group of people.

- Although two or three acronyms per manuscript may help to shorten it, particularly if they are in common use, resist the urge to reduce perfectly acceptable phrases into acronyms. Acronyms with more than four letters must be avoided. The best acronyms are pronounceable. Provide the full form of an acronym the first time it is used.

- An ideal sentence is short, with no more than two verbs and three punctuation marks. Put every verb and its subject noun close to each other. A pronoun is interpreted as the nearest preceding noun. Pay attention to number and tense.

- Do not often split infinitives. Never use dangling modifiers. Avoid an excess of superlatives and intensifiers.

- The practice of elegant variation, commonly taught in creative-writing courses, must be avoided. Use only one word for an entity or action, and imply just one entity or action by one word, throughout the manuscript. There are no exact synonyms. In any given context, there will be one and only one best word, which you must find and use.

- Consult a dictionary when you wish to use a word that is not part of your working vocabulary. Avoid fad words and phrases such as "paradigm", "heuristic", and "tipping point".

- Punctuate properly. Commas, semi-colons, and colons have different uses, and must not be used interchangeably. Use quotation marks only for direct quotations; otherwise, simply italicize. Exclamation marks must be used only for exclamations, not to highlight a surprising result. Use "And" to begin a sentence very rarely. Do not use contractions such as "haven't" and "wasn't".

- Show your enthusiasm by using the active voice, although the passive voice may be adopted carefully for a few sentences in order to create a specific effect. Often, a sentence becomes more forceful when an adjective is eliminated in favor of a related verb.

- Do not avoid incidental humor or wordplay, but make sure that such constructions are not obscure.

- Do not be verbose. Do not lose focus. Come to the point as soon as possible, so that the reader's valuable time is not wasted. 
- Do not be unnecessarily terse either. Imagine that your paper is being read by a firstyear graduate student. Put yourself in that reader's shoes and evaluate the intelligibility of your manuscript.

- Resist the temptation to write: "It can be easily shown that.....", or "It is trivial/obvious to show that...". Also, avoid the adjective "well-known". Such constructions are either superfluous or insulting to the reader. Do not use the adjective "so-called", except pejoratively. Use "respectively" sparingly. Do not use "model" and "system" in more than one context each in your manuscript.

- All items in a list must belong to the same part of speech; e.g., all must be nouns, or all must be adjectives.

- Define every symbol when it is first used in the manuscript. Make a table of all mathematical symbols and their meanings that you use in the manuscript. Use this table to ensure that only one symbol is used for a quantity. (This table will not be a part of your manuscript for most journals, but it will help you keep your notation straight.) Thus, do not use the symbol $i$ both as an index with integer values and to stand for $\sqrt{-1}$.

- Your notation should be such that scalars, vectors, and tensors must be clearly distinguishable from one another. Provide units for every quantity with units. SI units must be used.

- Number every mathematical expression that you set apart from the running text.

- A picture is worth a thousand words. Do not avoid putting schematics, graphs, and other types of diagrams to explain your work. But, every figure must be highly relevant to your manuscript. All lettering should be in sufficiently large characters to be legible.

- Do not provide complicated figures that only you can understand easily. Often dense 3D graphs are confusing to the reader; consider replacing a dense 3D graph by a few 2D graphs that bring out significant relationships.

- Figure captions and legends must be descriptive enough that every figure can be easily understood.

- If you reproduce a figure from a published work, cite the source; in addition, you may have to obtain copyright permissions from the author(s) and the publisher of the source.

- Do not expect reviewers to conduct literature searches on your behalf and to provide missing references.

- Never copy a citation from some other paper; indeed, never cite a publication whose hardcopy or electronic copy you do not possess. Do not cite very old papers unless you wish to point to something specific in that paper. Do not cite materials that are not available easily, such as internal memorandums and reports of a company.

- When you cite a book for a specific experimental fact, theoretical proof, etc., include the relevant page numbers from that book.

- Before submitting your manuscript for publication, have it read by a colleague just as I had Craig F. Bohren of Penn State read this manual and suggest changes and improvements.

Format your manuscript properly. Read and carefully follow the instructions available online at http://spie.org/x3655.xml for Journal of Nanophotonics authors. 